\title{
Proton electromagnetic form factors: present status and future perspectives at PANDA
}

\author{
E. Tomasi-Gustafsson ${ }^{1,2, a}$, on behalf of the PANDA Collaboration \\ ${ }^{1}$ CEA, IRFU, SPhN, Saclay, 91191 Gif-sur-Yvette Cedex, France \\ ${ }^{2}$ CNRS/IN2P3, Institut de Physique Nucléaire, UMR 8608, 91406 Orsay, France
}

\begin{abstract}
Data and models on electromagnetic proton form factors are reviewed, highlighting the contribution foreseen by the PANDA collaboration. Electromagnetic hadron form factors contain essential information on the internal structure of hadrons. Precise and surprising data have been obtained at electron accelerators, applying the polarization method in electron-proton elastic scattering. At electron-positron colliders, using initial state radiation, BABAR measured proton time-like form factors in a wide time-like kinematical region and the BESIII collaboration will measure very precisely proton and neutron form factors in the threshold region. In the next future an antiproton beam with momentum up to $15 \mathrm{GeV} / \mathrm{c}$ will be available at FAIR (Darmstadt). Measurements of the reaction $\bar{p}+p \rightarrow e^{+}+e^{-}$by the PANDA collaboration will contribute to the individual determination of electric and magnetic form factors in the time-like region of momentum transfer squared, as well as to their first determination in the unphysical region (below the kinematical threshold), through the reaction $\bar{p}+p \rightarrow e^{+}+e^{-}+\pi^{0}$. From the discussion on feasibility studies at PANDA, we focus on the consequences of such measurements in view of an unified description of form factors in the full kinematical region. We present models which have the necessary analytical requirements and apply to the data in the whole kinematical region.
\end{abstract}

\section{Introduction}

In next future the PANDA (antiProton Annihilation at Darmstadt) collaboration [1] will make use of the antiproton beam of momentum up to $15 \mathrm{GeV} / \mathrm{c}$ at FAIR (Facility for Ions and Antiproton Research) [2] to study open problems related to non-perturbative Quantum Chromo Dynamics (QCD), the theory that drives the strong interaction. Presently more than 500 physicists from 67 institutions and 17 countries participate to the PANDA Collaboration.

PANDA is at the same time a fixed target experiment and an internal experiment inside the high energy storage ring (HESR) because those antiprotons which do not interact, recirculate in the ring. One of the advantages of this experiment is that the detector and the accelerator are built and optimized together for best performances.

\footnotetext{
a e-mail: etomasi@cea.fr
} 
The kinematical domain which will be covered by PANDA is often called the transition or intermediate energy domain and the field of research "hadron physics", as in this energy range one can explore the internal structure of the hadron.

Hadron physics is usually classified in three major fields: hadron spectroscopy, hadron structure and interaction of hadrons. The PANDA experiment will make advances in all these fields, through $\bar{p} p$ and $\bar{p} A$ reactions, using antiproton beams with center of mass energies between 2.2 and $5.5 \mathrm{GeV}$.

The kinematical region covered by PANDA is especially well suited for charmonium and open charm spectroscopy. Antiproton-proton annihilation will create a gluon-rich environment. The high resolution will be crucial for the search and the understanding of the properties of gluonic excitations, as glueball and hybrids. PANDA will reach the threshold for charm baryon production, and open the study for open charm associated production: a large sample of $D \bar{D}$ pairs will be produced.

Here we focus on the annihilation of $\bar{p} p$ into an electron pair through a virtual photon of momentum squared $q^{2}=s$ ( $s$ is the total energy squared) which gives access to the hadron electromagnetic vertex $\gamma^{*} \rightarrow \bar{p} p$ (which is equivalent to $\gamma^{*} \rightarrow \bar{p} p$, assuming charge invariance of the strong and electromagnetic interactions). In this case, $q^{2}>0$, and this reaction (as well as the time reverse $e^{+}+e^{-} \rightarrow \bar{p}+p$ ) gives access to the time-like (TL) region of momentum transfer squared. These annihilation reactions are related through crossing symmetry to electron proton $(e p)$ elastic scattering, which scans the region of space-like (SL) momenta $\left(q^{2}=-Q^{2}<0\right)$.

\section{Proton form factors: experimental status}

An overview of selected data and models on proton form factors (FFs) is given in Fig. 1. Below we give a description of the experiments and the models, following the $q^{2}$ axis, highlighting the physics content of the different kinematical regions.

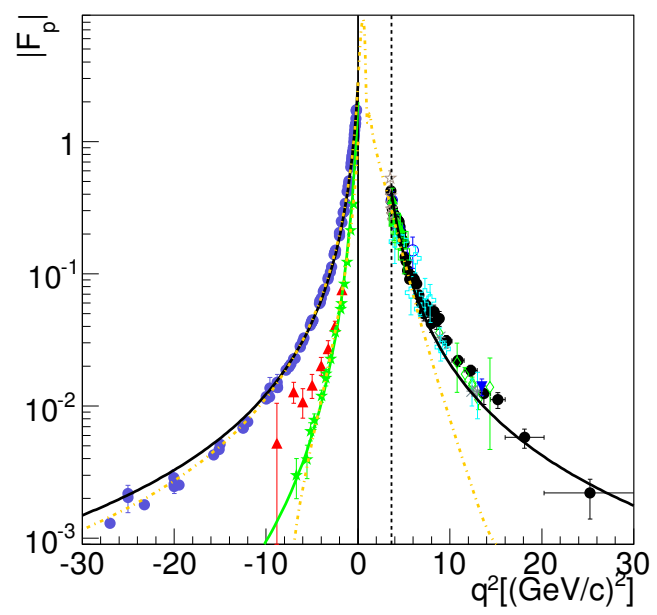

Figure 1. World data on proton FFs as a function of $q^{2}$. SL region: $G_{M}$ data (blue circles), $G_{E}$ data (red triangles) from unpolarized measurements [3] and from polarization measurements (green stars) [4]. The solid, green (black) line is the model prediction of Ref. [6] for $G_{E}\left(G_{M}\right)$. TL region: $\left|G_{E}\right|=\left|G_{M}\right|$ world data for $q^{2}>4 M_{p}^{2}$ and model prediction (black, solid line) for $G_{M}$ from Ref. [6] (dipole function). The orange, dash-dotted line is the prediction from Ref. [7]. 


\subsection{The space-like region: $q^{2} \leq 0$}

Traditionally ep elastic scattering is considered as the preferred way to investigate the internal structure of the proton.

An elegant formalism was built on the assumption that the electron-hadron interaction occurs through the exchange of a virtual photon of four-momentum $q$.

In this case, the cross section for electron hadron elastic interaction has a characteristic dependence on $\cot ^{2} \theta$ ( $\theta$ is the electron scattering angle). This is a consequence of the one-photon exchange mechanism and it is based on the $J^{P C}=1^{--}$nature of the virtual photon.

The electromagnetic vertex $\gamma^{*} \rightarrow h h$ ( $h$ is any hadron) is defined by two structure functions, which, in turn, are expressed in terms of $(2 S+1)$ FFs, $S$ being the hadron spin, assuming parity and time-invariance. FFs are analytical functions of only one variable, $q^{2}$. Protons (and neutrons) have two FFs, electric $G_{E}$, and magnetic $G_{M}$, which are normalized at $q^{2}=0$ to the static values of the charge $G_{E}(0)=1$ and of the magnetic moment, $G_{M}(0)=\mu_{p}$.

The particular angular dependence of the differential $e N$-cross section is at the basis of the method to determine both nucleon electromagnetic FFs, defining a reduced cross section $\sigma_{\text {red }}$, which is linear in the variable $\varepsilon=\left[1+2(1+\tau) \tan ^{2}\left(\theta_{e} / 2\right)\right]^{-1}\left(\theta_{e}\right.$ is the electron scattering angle in the laboratory system)

$$
\sigma_{\mathrm{red}}\left(\theta_{e}, Q^{2}\right)=\left[1+2 \frac{E}{M} \sin ^{2}\left(\theta_{e} / 2\right)\right] \frac{4 E^{2} \sin ^{4}\left(\theta_{e} / 2\right)}{\alpha^{2} \cos ^{2}\left(\theta_{e} / 2\right)} \times(1+\tau) \varepsilon \frac{d \sigma}{d \Omega}=\tau G_{M}^{2}+\varepsilon G_{E}^{2},
$$

where $E$ is the beam energy, $\tau=Q^{2} / M^{2}$, and $M$ is the proton mass. The measurement of the differential cross section at fixed $Q^{2}$, for different angles allows to extract the electric and magnetic FFs as the slope and the intercept, respectively, of this linear distribution. This is called the Rosenbluth method [9]. One can see that

- the backward $e N$-scattering $\left(\theta_{e}=\pi, \varepsilon=0\right)$ is determined by the magnetic FF only. The slope for $\sigma_{\text {red }}(\varepsilon)$ is sensitive to $G_{E}^{2}$;

- the contribution of the magnetic term is weighted by the factor $\tau$ which increases as $Q^{2}$ increases;

- the (unpolarized) cross section contains FFs squared, being insensitive to their sign.

Since the pioneering experiments of Hofstadter [10] several measurements of the unpolarized cross section for $e p$ elastic scattering have been performed, the determination of $G_{E}$ becoming imprecise as $Q^{2}$ increases for the reasons given above. Moreover, radiative corrections, which depend on $\varepsilon$ and $Q^{2}$ too, become larger with $Q^{2}$, reaching up to $50 \%$. They have been generally applied at first order $\left(\alpha^{3}\right)$ in the electromagnetic fine constant $\alpha$.

From unpolarized cross section measurements the determination of $G_{E}$ and $G_{M}$ has been done up to $Q^{2} \simeq 8.8 \mathrm{GeV}^{2}$ [3] and $G_{M}$ has been extracted up to $Q^{2} \simeq 31 \mathrm{GeV}^{2}$ [11] under the assumption that the electric FF vanishes $\left(G_{E}=0\right)$ or that it equals the magnetic FF, $G_{M}$, scaled by the proton magnetic moment $G_{E}=G_{M} / \mu_{p}$. In Fig. 2 the magnetic moment is divided by the dipole function: $G_{D}=\left[1-Q^{2} / O .71\right]^{-2}$.

Polarization phenomena were studied and developed by the Kharkov school since the mid of last century. In Refs. [12,13] it was first pointed out that the polarized cross section contains the interference of the amplitudes, giving access to the sign of FFs and being more sensitive to a small $G_{E}$ contribution.

The polarization method, which requires a longitudinally polarized electron beam and the measurement of the polarization of the scattered proton, could only recently be applied, due to the availability of high intensity, highly polarized electron beams, and proton polarimetry in the $\mathrm{GeV}$ range. It 
was used in particular by the JLab GEp collaboration in a series of experiments, measuring precisely the ratio of the electric to magnetic FFs up to $Q^{2}=8.9 \mathrm{GeV}^{2}$ [4] which is directly related to the ratio of the transverse over longitudinal polarization in the scattering plane of the recoil proton.

The measurement of the FFs ratio gave very precise results, as it was expected, because, at first order, the beam helicity as well as the analyzing power of the proton polarimeter cancel, reducing the systematic errors. But the experiment showed also a surprising behavior: a monotone decreasing of the FFs ratio when $Q^{2}$ increases. An extrapolation of this tendency at large $Q^{2}$ may lead to the ratio passing through zero and even becoming negative. As $G_{M}$ is supposed to be well known from the unpolarized cross section (as the magnetic term dominates at large $Q^{2}$ ), the present understanding is that $G_{M}$ follows a dipole ( $Q^{-4}$ ) behavior and that the electric FF follows a steeper decreasing (Fig. 3).

The present situation is that unpolarized experiments give a FFs ratio consistent with unity (with a larger error as $Q^{2}$ increases) whereas polarized experiments deviates from unity as $Q^{2}$ increases.

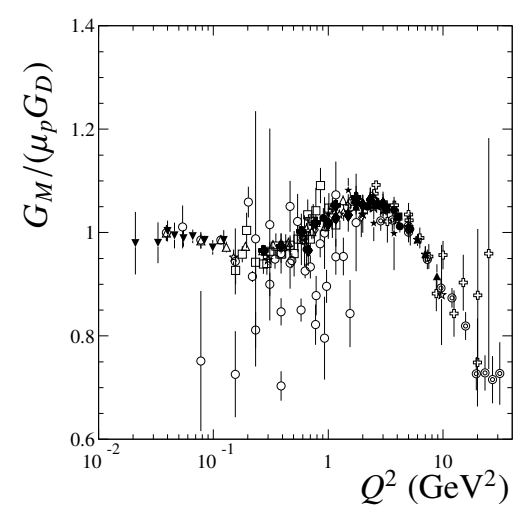

Figure 2. World data on $G_{M} / \mu_{p} G_{D}$ as a function of $Q^{2}$.

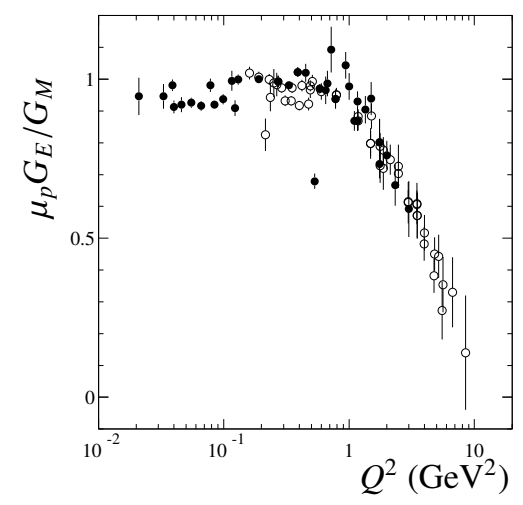

Figure 3. Ratio $\mu_{p} G_{E} / G_{M}$ from polarization data (empty symbols) and from selected unpolarized cross section data (solid symbols) 
The reason has likely to be attributed to the contribution of higher order radiative corrections (for a recent discussion see Ref. [5]). Note that unpolarized data, selected in experiments where radiative corrections did not exceed 20\%, also show a deviation of the ratio from unity (Fig. 3).

It is expected that data on individual FFs in the TL region will help to clarify this issue.

\subsection{The low $Q^{2}$ region}

The precision on the measurement of FFs in the low $q^{2}$ region is important at least in two respects:

- the determination of axial and strange FFs relies on the precise knowledge of the electromagnetic FFs;

- the root mean squared charge radius is related to the derivative of the FF at $Q^{2}=0$ :

$$
<r_{c}^{2}>=-\left.6 \frac{d G_{E}\left(Q^{2}\right)}{d Q^{2}}\right|_{Q^{2}=0} .
$$

The problem of the proton size has been recently object of large interest due to experiments on muonic hydrogen by laser spectroscopy measurement of the $v p(2 \mathrm{~S}-2 \mathrm{P})$ transition frequency [14]. The result on the proton charge radius obtained in this experiment is one order of magnitude more precise and smaller by seven standard deviation compared to the best value previously obtained $r_{c}=0.8768(69)$ fm (CODATA) [15]. This determination was based on hydrogen spectroscopy, which is more precise than, but compatible with, electron proton elastic scattering at small values of the four momentum transfer squared. Such discrepancy stimulated theoretical and experimental efforts, in particular precise measurements of ep elastic scattering, at low $Q^{2}$ [16] and new experiments with muon beams [17]. Experiments are quite difficult, because, being strongly dependent on the slope of the FFs at $q^{2}=0$ where they are constrained by the static value, they require measurements of the cross section at very small transferred momenta, which are in principle impossible to obtain with infinite precision. Hence, the usual procedure to determine such radius consists in fitting the low- $Q^{2}$ data and taking the analytic derivative at $q^{2}=0$ of the fit function. It is interesting to notice that the mean square radius is proportional to the logarithmic derivative of the corresponding FF in the origin and hence does not depend on the normalization.

\section{The time-like region: $q^{2} \geq 4 M^{2}$}

The positive $q^{2}$ region, above the kinematical threshold $q^{2}=4 M^{2}$ can be accessed through the annihilation reaction $\bar{p}+p \rightarrow e^{+}+e^{-}$into a virtual photon which decays into a lepton pair. Electromagnetic proton FFs can be measured through a precise measurement of the angular distribution of one of the outgoing leptons. The expression of the differential cross section for $\bar{p}+p \rightarrow e^{+}+e^{-}$is [18]

$$
\frac{d \sigma}{d \cos \theta}=\frac{\pi \alpha^{2}}{2 \beta q^{2}}\left[\left(1+\cos ^{2} \theta\right)\left|G_{M}\right|^{2}+\frac{1}{\tau} \sin ^{2} \theta\left|G_{E}\right|^{2}\right] ; \beta=\sqrt{1-\frac{4 M^{2}}{q^{2}}}
$$

FFs are complex in TL region, and their moduli enter in this expression. As in SL region, no interference appears, and the magnetic term is enhanced by the factor $\tau$ at large $q^{2}$. Unlike in the SL region, the differential cross section gives access to the full information on the proton FFs in a single experimental measurement. The characteristic $\cos ^{2} \theta$ dependence $(\theta$ is the CMS angle of the outgoing lepton) is a signature of the one-photon exchange mechanism ${ }^{1}$.

\footnotetext{
${ }^{1}$ The even dependence on $\cos \theta$ in TL region corresponds to the linearity in $\cot ^{2} \theta_{e}$ in SL region, as it can be shown using crossing symmetry [19].
} 
The time reverse reaction $e^{+}+e^{-} \rightarrow \bar{p}+p$ brings the same physical information. In order to scan a large region of $q^{2}$, the process $e^{+}+e^{-} \rightarrow \bar{p}+p+\gamma$ can be used when the emitted photon is hard. When the electron remains on shell after the photon emission, the cross section can be factorized in a radiator function (a term which depends on the energy and the angle of the hard photon) and in the cross section for the process of interest $e^{+}+e^{-} \rightarrow \bar{p}+p$ (initial state radiation, ISR).

The individual determination of FFs in the TL region has not yet been done due to the limitation in the intensity of antiproton beams or the luminosity of $e^{+} e^{-}$colliders which did not allow a precise and complete measurement of the angular distribution of the outgoing leptons.

The results are often given in terms of an effective FF derived from the total (or integrated) cross section under the assumption $G_{E}=G_{M}=G_{\text {eff }}$ :

$$
G_{\text {eff }}=\sqrt{\frac{\left|G_{E}\right|^{2}+2 \tau\left|G_{M}\right|^{2}}{1+2 \tau}} .
$$

or the equivalent form corrected by the angular range, if limited.

At the moment the best data are the ones achieved by BaBar [22, 23] see Fig. 4. For the complete set of references the reader is referred to [24]. The data on $\left|G_{\text {eff }}\right|$ show several structures, superimposed to a general trend which follows the dipole behavior.
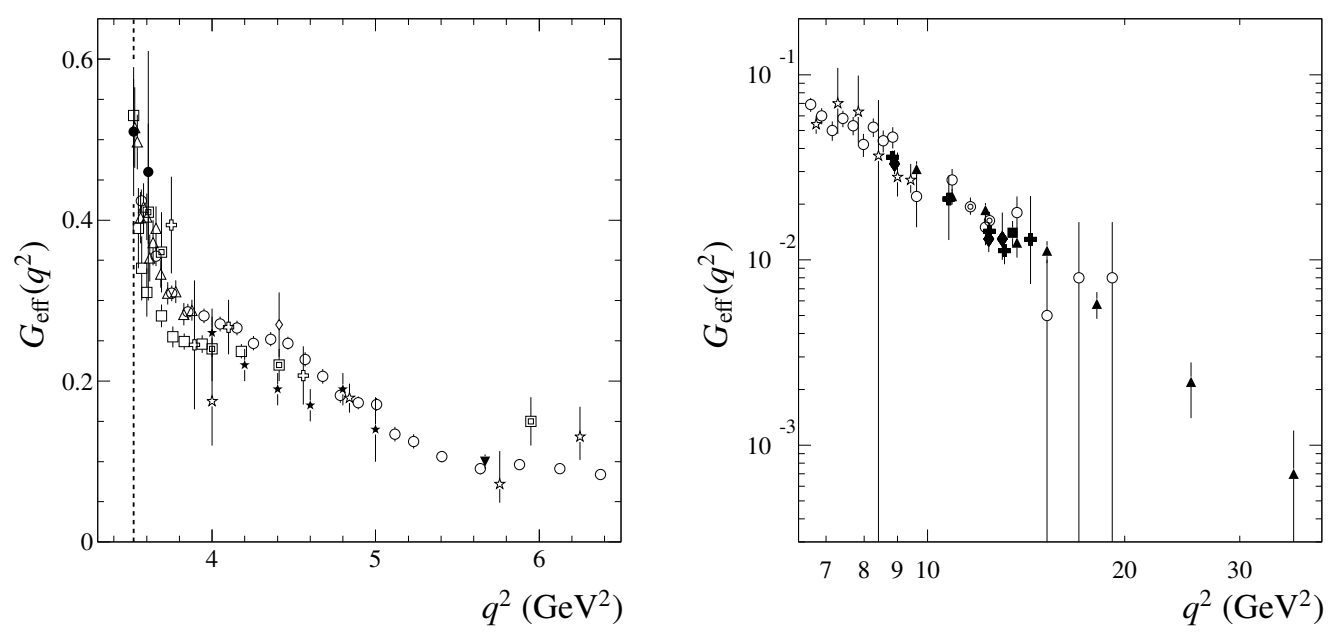

Figure 4. World data of the effective proton $\mathrm{FF}, G_{\text {eff }}\left(q^{2}\right)$, as a function of the momentum transfer squared, in the low (left) and high (right) momentum transfer regions. The data from Babar, are shown as open circles Refs. [22] and triangles [23].

\subsection{The threshold region}

The threshold region is particularly intriguing. Several experiments have been performed, in the near threshold region, with increasing precision. A flat behavior is observed near threshold.

At threshold it is expected that only the $S$-wave plays a role, and $\left|G_{E}^{p}\left(4 M_{p}^{2}\right)\right|=\left|G_{M}^{p}\left(4 M_{p}^{2}\right)\right|$. It has been observed that the presence of the Coulomb factor, plays a specific role compensating the phase-space relative velocity. The extrapolation of the experimental cross section up to the threshold gives:

$$
\sigma\left(e^{+} e^{-} \rightarrow \bar{p} p\right)\left(4 M^{2}\right)=(0.85 \mathrm{nb})\left|G\left(4 M^{2}\right)\right|^{2},
$$


where $G\left(4 M_{p}^{2}\right)$ is the common value of the electric and magnetic FF at threshold. Introducing the experimental value of the cross section, one finds $G\left(4 M_{p}^{2}\right)=1$, like in the case of a pointlike fermion [25].

\subsection{The PANDA contribution}

The results presented in this section are referenced in the $\mathrm{PhD}$ thesis of Ref. [27] and are being finalized in a forecoming publication.

PANDA will contribute to the field of FFs in the TL region at least in three respects:

- the electric and magnetic FFs will be measured separately for the first time, in a wide kinematical range;

- the cross section measurement will allow the extraction of the effective FF below $q^{2}=30 \mathrm{GeV}^{2}$;

- the first measurement below the kinematical threshold will be performed.

With respect to previous experiments with antiproton beams, PANDA will have higher luminosity (up to $2 \cdot 10^{32} \mathrm{~cm}^{-2} \mathrm{~s}^{-1}$, better beam momentum resolution (up to $\Delta p / p \sim 10^{-5}$ ), larger coverage of the solid angle, more precise electromagnetic calorimetry and better particle identification for $\pi, K, e$, $\mu, p$.

In order to gather all the necessary information from the antiproton-proton collisions, the PANDA detector has to be able to provide precise trajectory reconstruction, energy and momentum determination and has to be very efficient in identifying charged particles as well as photons, in a wide kinematical range, in an environment where the average reaction rate is expected to reach up to 20 $\mathrm{MHz}$.

Of course, PANDA takes advantage of the experience of previously built detectors, but has to afford challenges sometimes comparable or exceeding those of LHC detectors. For example, the electromagnetic (EM) calorimeter, consisting of Lead Tungstate $\mathrm{PbWO}_{4}$ crystals, will insure an efficient photon detection from $10 \mathrm{MeV}$ to $10 \mathrm{GeV}$. A similar type of crystals has been previously used, for example by the CMS experiment at the LHC. However, the photon detection in a high luminosity environment and within such a large energy range is a specific requirement of PANDA compared to previous experiments and demands new solutions for detectors, electronics, and data acquisition. In particular, a low energy threshold of $3 \mathrm{MeV}$ for an individual detector module requires the maximization of the $\mathrm{PbWO}_{4}$ light output [20] and the light detection efficiency. The PANDA EMC calorimeter will be operated at a temperature of $\mathrm{T}=-25^{\circ} \mathrm{C}$ to take advantage of the increase of the scintillation light by a factor of four. This requires the proper operation of all components in a low temperature environment.

The main challenge of the measurement of the reaction $\bar{p}+p \rightarrow e^{+}+e^{-}$is the identification of the lepton pair in a huge hadronic background, in particular the production cross section of a charged pion pair, which is larger than the signal by six orders of magnitude and has very similar kinematics. On the other hand, the kinematical selection helps for the suppression of hadronic channels with more than two particles in final state, and of secondary particles.

The simulation, the reconstruction and the analysis have been fully performed for three values of $s=q^{2}=5.4,8.2$ and $13.9 \mathrm{GeV}^{2}$, using the PANDARoot software for both channels, lepton and pion pair production [27]. The first step of the analysis is the selection of the best back-to-back pair in the center of mass system. Then, the reconstructed kinematical variables and particle identification probabilities are built and the best cuts, which can suppress the pion background keeping the best efficiency for the signal, are set. 
Using the efficiency curve as in Fig. 5, the error on the FF ratio can be extracted. Fig. 6 summarizes the present situation. The projections for PANDA according to different models are shown in Fig. 6.

The simulated data have been reported along the models, to show the dependence of the estimated (statistical only) error on the value of $R$. The statistical error will certainly allow to discriminate among the predictions available today which are reported on the figure.

Note that the models reproduce qualitatively well the existing data in SL region $\left(G_{E}\right.$ and $G_{M}$ for proton and neutron) and $G_{\text {eff }}$ in TL region, however the predictions for $G_{E}$ and hence for the ratio $R=G_{E} / G_{M}$ as well as for polarization observables are very different [26].

With the luminosity known at a level of a few percent, PANDA may first determine $G_{E}$ and $G_{M}$ separately, for intermediate values of $q^{2}$.

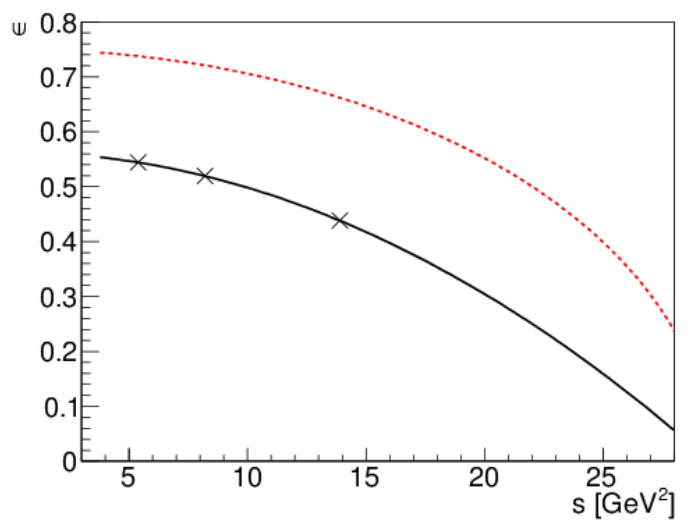

Figure 5. Simulated efficiency $\epsilon$ as function of $q^{2}$, for the PANDA experiment, done at three kinematics $q^{2}=5.4$, 8.2 and $13.9 \mathrm{GeV}^{2}$ (crosses) from Ref. [27]. The solid line is an inter(extra)polation of the points. The dashed line represents the square root of the efficiency, the quantity which enters in the experimental error.

These data, compared to the corresponding information obtained in electron proton elastic scattering experiments, will constitute a stringent test of the asymptotic behavior predicted by QCD and of analytical properties of elastic amplitudes. There is indication that the asymptotic region, where SL and TL values are expected to converge following analyticity, is reached at $Q^{2} \sim 30 \mathrm{GeV}^{2}$. PANDA will investigate more precisely this region.

\section{The unphysical region: $0 \leq q^{2} \leq 4 M^{2}$}

The detection of a pion accompanying the lepton pair, $\bar{p}+p \rightarrow e^{+}+e^{-}+\pi^{0}$ will allow to investigate for the first time the "unphysical region" below the $\bar{p} p$ kinematical threshold. No data are available yet in this kinematical region where a huge cross section is expected due to resonant structures. The scattering mechanism through the exchange of a (anti)proton of low virtuality gives access to proton(neutron) FFs below the kinematical threshold, the neutral(negative) emitted pion carries the excess of energy. This mechanism was first suggested in Ref. [32] on the time reverse reaction $\pi^{-}+p \rightarrow n+e^{+}+e^{-}$. More recently the cross section was calculated in the kinematical domain relevant to PANDA, Ref. [33], a model independent calculation of all observables was done in terms 


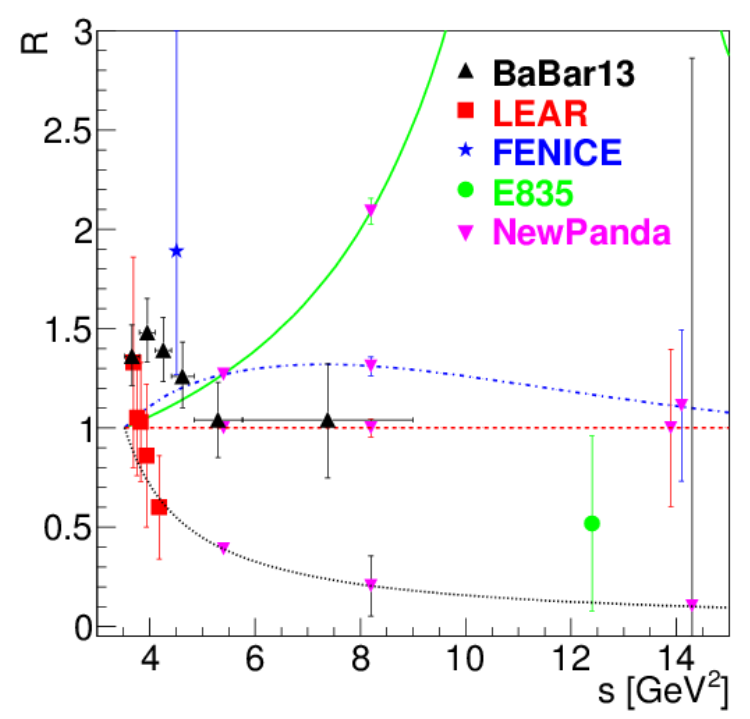

Figure 6. Data on FFs ratio [27], data are from: Ref. [22] (empty circles), Ref. [31] (empty squares), Ref. [21] (empty triangles). The simulated data for PANDA (solid circles) have been reported along the prediction of different models for the TL ratio: [28, 29] $\left(R_{p}=1\right.$, solid line), [7] (dashed line), [30] (dash-dotted line), [6] (dotted line).

of FFs in Ref. [34]. More precisely, it was shown in Ref. [33] that the contribution of the $t$-channel can be parameterized in terms of two unknown functions $F_{1,2}(t, u)$ which depend of both variables $t$ and $u\left(t\right.$ and $u$ are the Mandelstam variables, $\left.s+t+u=2 M^{2}\right)$ and rapidly decrease in the region $s \sim|t| \sim|u|$ as $F_{1,2}(t, u) \sim\left(M^{2} / s\right)^{n} \ll 1$ where $n>1$. Let us note that in the limits $|t| \ll s$ or $|u| \ll s$ these quantities do coincide with the electromagnetic FFs of the proton. In these kinematical regions the Regge-factors have to be included into the differential cross section. These factors may strongly suppress the cross section.

In the region $|t| \sim|u| \sim s \gg M_{p}^{2}$ these amplitudes are unknown functions of both kinematical variables. They have the form $\left(M_{p}^{2} / s\right)^{n} \phi(t / s)$, where the exponent $n$ is determined by quark-counting rules and are not related with the Dirac and Pauli FFs of the proton. A similar behavior is expected for the vertex of pion interaction with nucleons. Intermediate states such as nucleonium ( $p \bar{p}$ bound states), vector and scalar mesons (including radially excited meson states) can in principle contribute. It is known that the largest anomalous vertex is $\rho \rightarrow \omega \pi$, because it has the largest quark coupling. The interaction of the vector $\omega$-meson with the nucleus in the vertex $\omega \rightarrow p p$ contains information on the strong proton and meson couplings. It can be described in terms of TL proton vector FFs, which have, in principle, complex nature. The vertex $\omega \rightarrow \pi \gamma^{*}$ can be described either by a phenomenological parametrization, or through a triangle vertex, which is calculable in the frame of Nambu-Jona-Lasinio model and relevant also to the transition pion FF. Predictions for angular distributions and cross sections can be found in Ref. [35].

The reaction $\bar{p}+p \rightarrow e^{+}+e^{-}+\pi^{0}$ has also been investigated in backward kinematics, with the aim to get information of transition distribution amplitudes in Ref. [36] and in the frame of the Generalized 
Distributions Amplitudes approach in Ref. [37]. The Regge behavior of the scattering amplitude and the resonant character of the annihilation amplitude were not considered in those papers.

PANDA will be able to investigate this process. As for the reaction $\bar{p}+p \rightarrow e^{+}+e^{-}$, hadronic background constitutes an experimental challenge, in particular three pion production, for which no reliable model is available in the relevant kinematical range. The data on few pion production will also be collected by PANDA and the information from the data themselves will be used for the background suppression. These contain by themselves interesting information on the reaction mechanism and test QCD predictions.

\section{Nucleon Models}

The dipole behavior of FFs can be understood in terms of scaling rules predicted by QCD, assuming that the virtual photon transfers the momentum to each constituent quark through one gluon exchange, leaving the proton in its ground state $[28,29]$. It can be also understood in the Breit frame or in nonrelativistic approximation, where FFs are Fourier transform of the charge and magnetic distributions. The dipole form would correspond to an exponential distribution of these distributions.

Since the data based on the Akhiezer-Rekalo method appeared, a number of theoretical papers have been published. Most of the nucleon models had to be revised to account for the deviation of the ratio from unity. If few models had predicted the FF ratio decreasing, as the soliton model, the di-quark model and in particular the vector dominance model of Refs. [7, 8], not all these models reproduced well all four nucleon FFs, electric and magnetic, for proton and neutron. Moreover, the description of light nuclei as Deuteron or Helium, relies on the description of the constituent nucleons, and the remaining corrections (such as relativistic corrections, meson exchange currents, or isobar contributions) have been often adjusted to reproduce the data.

Dispersion relations (DR) represent a powerful theoretical tool which exploits the analytic properties of form s. FFs are defined for real values of $q^{2}$, but they can be analytically continued into the $q^{2}$-complex plane, apart from a cut along positive $q^{2}\left(4 m_{\pi}^{2}, \infty\right)$. They are bounded functions for all finite values of $q^{2}$. The complex poles, representing the resonant contributions, must lie in the so-called unphysical sheets, in the secondary Riemann planes, otherwise they would spoil the analyticity.

They provide almost model-independent results and parametrizations that, mainly due to the analytic treatment, are naturally valid in all kinematical regions and, in principle, in the whole complex plane. They are strongly constrained by the data. This constitutes also a limitation because only relative phases are experimentally observable. Moreover, as the integration in the DR is performed all over the infinite cut, not all TL $q^{2}$ are experimentally accessible, in particular in the unphysical region, where the main contributions from intermediate states is expected.

Recently a model was suggested to interpret nucleon electromagnetic FFs both in SL and TL regions [6]. It assumes that in the $e p$ collision or in the $e^{+}+e^{-} \leftrightarrow \bar{p}+p$ annihilation a large quantity of energy (mass) and momentum is concentrated in a small volume creating a strong gluonic field, i.e., a gluonic condensate of clusters with a randomly oriented chromo-magnetic field. This condition generates an effect similar to the screening of a charge in plasma. Applied to the scalar part of the field, it explains the additional suppression of the electric FF, and leaves unchanged the predictions from quark counting rules for the magnetic FF.

Similarly, in TL region, above the physical threshold, $q^{2} \geq 4 M_{p}^{2}$, the vacuum state created at the collision, transfers all the energy to a S-wave state with total spin 1, consisting in at least six massless valence quarks, a set of gluons and a sea of current $q \bar{q}$ quarks, with total energy $q_{0}>2 M_{p}$, and total angular momentum equals to unity. The quarks as partons have no structure $\left(\left|G_{E}\right|=\left|G_{M}\right|=1\right)$, which may explain the point-like behavior of FFs at threshold and is consistent with observation. Then, 
the current quarks (antiquarks) absorb gluons and transform into constituent quarks (antiquarks). For the same reasons as in SL region, also in TL region an additional suppression of the electric FF is expected.

This model has one free parameter, in principle calculable, and it is expected to apply starting from moderate values of the momentum transfer. It gives a good description of FFs in the whole kinematical range. The results are illustrated in Fig. 1, compared to the data and to the model of Ref. [7].

\section{Future perspectives and Conclusions}

The field of proton electromagnetic FFs is very active, experimentally and theoretically. Advances are expected in the next future.

Polarization played a major role in SL region, allowing a precise information on the electric FF. If available at antiproton facilities, it would also bring unique information in TL region. A high energy polarized antiproton beam was obtained from the parity violating decay of $\bar{\Lambda}^{0}$ hyperons at the Tevatron. Although the intensity was limited to $10^{5}$ antiprotons per second, physics experiments were carried out. Let us stress that in TL region single spin polarization observables determine the phase of FFs, which is a very sensitive quantity to be compared to the nucleon models.

PANDA is expected to give essential contributions to FFs in the TL region, concerning the individual measurement of the electric and magnetic FFs in the intermediate $Q^{2}$ region, the annihilation cross section into a lepton pair at large values of momentum transfer squared, and unique information in the unphysical region.

The threshold region will be further investigated, at electron-positron facilities, by BESIII at BEPCII, CMD3 and SND at VEPP2000, using the ISR method, and/or a detail scan of the threshold region. Moreover neutron TL FFs will also be measured and compared to the four existing points measured at FENICE. Note that these points are two times larger than the proton TL FF at the same $q^{2}$ value.

In SL region MAMI at Mainz will continue the programme of precise FFs measurements at low $Q^{2}$. At JLab, after the energy upgrade at $11 \mathrm{GeV}$, experimental programs on all four nucleon FFs with the high quality (polarized) electron beams are planned. The data will cover a wider kinematical domain with increased precision.

Approaches as the soliton models, constituent quark model, or conformal field theories have been recently applied to the TL region. Other phenomenological concepts as quark-hadron duality or generalized parton distributions have been developed during the recent years and applied to FF data:

DR are a powerful tool to connect the real and imaginary part of FFs, considered analytical functions. An important step will be reached when data in the unphysical region will serve as input to these models.

The theoretical effort will be focused on a global understanding of annihilation and scattering reactions in order to have a coherent picture of the nucleon for all values of momentum transfer square.

\section{Acknowledgments}

The review of FFs is largely based on the collection of data and on the work of Ref. [24] realized with S. Pacetti and R. Baldini, who are warmly acknowledged for interesting discussions and close collaboration. Thanks are due to D. Marchand for careful reading of the manuscript. 


\section{References}

[1] The PANDA Experiment website, URL: http://www-panda.gsi.de

[2] The new large scale accelerator facility in Europe website, URL: http://www.fair-center.eu

[3] L. Andivahis, et al., Phys.Rev. D 50, 5491 (1994).

[4] A. Puckett, et al., Phys.Rev.Lett. 104, 242301 (2010).

[5] E. Kuraev, A. Ahmadov, Y. M. Bystritskiy, E. Tomasi-Gustafsson, Phys.Rev. C 89, 065207 (2014).

[6] E. Kuraev, E. Tomasi-Gustafsson, A. Dbeyssi, Phys.Lett. B 712, 240 (2012).

[7] F. Iachello, Q. Wan, Phys.Rev. C 69, 055204 (2004).

[8] F. Iachello, A. Jackson, A. Lande, Phys.Lett. B 43, 191 (1973).

[9] M. Rosenbluth, Phys.Rev. 79, 615 (1950).

[10] R. Hofstadter, F. Bumiller, M. Croissiaux, Phys.Rev.Lett. 5, 263 (1960).

[11] R. Arnold, P. E. Bosted, C. Chang, J. Gomez, A. Katramatou, et al., Phys.Rev.Lett. 57, 174 (1986).

[12] A. Akhiezer, M. Rekalo, Sov.Phys.Dokl. 13, 572 (1968).

[13] A. Akhiezer, M. Rekalo, Sov.J.Part.Nucl., 4, 277 (1974).

[14] A. Antognini et al., Science 339, 417 (2013).

[15] P.J. Mohr,et al., Rev.Mod.Phys.",80,"633-730, (2008).

[16] J. C. Bernauer et al. [A1 Collaboration], Phys.Rev. C 90, 015206 (2014).

[17] R. Gilman et al. [MUSE Collaboration], arXiv:1303.2160 [nucl-ex].

[18] A. Zichichi, S. Berman, N. Cabibbo, R. Gatto, Nuovo Cim. 24, 170 (1962).

[19] M. P. Rekalo, E. Tomasi-Gustafsson, D. Prout, Phys.Rev. C 60 042202, (1999) .

[20] M. Kavatsyuk et al. [PANDA Collaboration], Nucl.Instrum.Meth. A 648, 77 (2011).

[21] R. Baldini, C. Bini, P. Gauzzi, M. Mirazita, M. Negrini and S. Pacetti, Eur.Phys. J. C 46, 421 (2006).

[22] J. Lees, et al., Phys.Rev. D 87, 092005 (2013).

[23] J. P. Lees et al. [BaBar Collaboration], Phys.Rev. D 88, 072009 (2013).

[24] S. Pacetti, R. Baldini-Ferroli and E. Tomasi-Gustafsson (2014), DOI: 10.1016/j.physrep.2014.09.005.

[25] R. Baldini, S. Pacetti, A. Zallo, A. Zichichi, Eur.Phys.J. A 39, 315 (2009).

[26] E. Tomasi-Gustafsson, F. Lacroix, C. Duterte, G. Gakh, Eur.Phys.J. A 24, 419 (2005).

[27] A. Dbeyssi, Ph.D. thesis, Université Paris-sud (2013).

[28] V. Matveev, R. Muradyan, A. Tavkhelidze, Teor.Mat.Fiz. 15, 332 (1973).

[29] S. J. Brodsky, G. R. Farrar, Phys.Rev.Lett. 31, 1153 (1973).

[30] E. L. Lomon, S. Pacetti, Phys.Rev. D85, 113004 (2012).

[31] G. Bardin, et al., Nucl.Phys. B 411, 3 (1994).

[32] M. P. Rekalo, Sov.J.Nucl.Phys. 1, 760 (1965).

[33] C. Adamuscin, E. Kuraev, E. Tomasi-Gustafsson, F. Maas, Phys.Rev. C 75, 045205 (2007).

[34] G. Gakh, A. Rekalo, E. Tomasi-Gustafsson, J. Boucher, A. Gakh, Phys.Rev. C 83, 025202 (2011).

[35] E. A. Kuraev, Y. M. Bystritskiy, V. V. Bytev, E. Tomasi-Gustafsson, A. Dbeyssi and E. TomasiGustafsson, J.Exp.Theor.Phys. 115, 93 (2012).

[36] B. Pire and L. Szymanowski, Phys. Lett. B 622, 83 (2005).

[37] P. Kroll and A. Schafer, Eur.Phys.J. A A26, 89 (2005). 